\title{
Factores pronósticos en ependimomas intramedulares
}

\author{
Enrique Orrego Puelles* 1,a; Katya Chávez Barboza ${ }^{2, b}$; Mary Heredia Estela 3,b
}

RESUMEN

Objetivo: Investigar los factores de buen pronóstico, la sobrevida global (SG) y la sobrevida libre de progresión (SLP) en un grupo de personas operadas de ependimoma intramedular. Los factores están relacionados con la persona, el tumor, el tipo de resección quirúrgica y el tratamiento complementario.

Materiales y métodos: Estudio retrospectivo, analítico y descriptivo de 40 pacientes operados de ependimoma intramedular entre los años 1985 y 2017 en el Instituto Nacional de Enfermedades Neoplásicas (INEN). Se usó el SPSS Windows 2017. Para calcular la sobrevida se empleó el producto de los límites de Kaplan Meier, y el test del logaritmo del rango para la comparación de curvas de sobrevida. La probabilidad (valor de $p$ ) para ser considerada significativa fue $p<0,05$.

Resultados: De los 40 casos, 33 pacientes fueron adultos y 7 niños; la relación hombre: mujer fue 1,4:1. El síntoma más frecuente fue déficit motor en $97,50 \%$ de los pacientes. La media y mediana del tiempo de enfermedad fue de 24,90 y 19 meses, con un rango de 2 a 108 meses. Las localizaciones más frecuentes fueron la región cervical (47,50 \%) y lumbar ( 25 \%). La siringomielia se presentó en el 40,00 \% de casos. Según el tamaño tumoral, el 60,00\% estuvo en los niveles 3 a 5 ; y el 32,50\% fue mayor de 5 niveles. El tipo de resección fue total en el $30 \%$ de las cirugías; subtotal, en el $60 \%$; y parcial, en el $10 \%$. La patología correspondió a grado II en el 77,50 \% de los casos; grado I, en el 15,00 \%; y grado III, en el 7,50\%. El 82,50\% recibió radioterapia (RT) y tres pacientes, quimioterapia (QT). La supervivencia general fue de 240 meses. La edad, sexo, tamaño del tumor y la patología no fueron estadísticamente significativos en la sobrevida. Con la resección total, la sobrevida a 5 años fue de 100,00\%; a 10 años, 90,00\%; y a los 15 años fue también de 90,00\%, con diferencias estadísticamente significativas en relación con resección subtotal y parcial $(p<0,001)$. También existió una diferencia estadísticamente significativa en el grupo de pacientes que recibieron radioterapia $(p<0,002)$. La sobrevida libre de progresión fue de 168 meses. Hubo diferencias estadísticamente significativas en la sobrevida libre de progresión con resección quirúrgica total y radioterapia adyuvante y no existieron tales diferencias con las otras variables examinadas. El déficit motor leve a moderado mejoró el pronóstico de vida en el postoperatorio y durante su seguimiento.

Conclusiones: Varios factores influyen en la sobrevida global y en la sobrevida libre de recurrencia: los más frecuentes son la resección total y la radioterapia; y el mejor estado de la función motriz en el preoperatorio, en menor proporción.

Palabras clave: Ependimoma; Intramedular; Radioterapia; Quimioterapia; Pronóstico (Fuente: DeCS BIREME).

\section{Prognostic factors in intramedullary ependymomas}

\author{
ABSTRACT
}

Objective: To study the good prognostic factors, overall survival (OS) and progression-free survival (PFS) in a group of patients who underwent an intramedullary ependymoma surgery. Such factors are related to the patient, tumor, type of surgical resection and adjunctive treatment.

Materials and methods: A retrospective, analytical and descriptive study conducted in 40 patients who underwent an intramedullary ependymoma surgery between 1985 and 2017 at the Instituto Nacional de Enfermedades Neoplásicas (INEN). The research used IBM SPSS Statistics for Windows 2017. The survival rate was estimated using the Kaplan-Meier product-limit model. The comparison of survival curves was determined by the log-rank test. A probability ( $p$ value) less than 0.05 was considered as statistically significant.

Results: Out of the 40 patients, 33 were adults and seven were children. The male to female ratio was 1.4:1. The most frequent symptom was motor deficit, which occurred in $97.50 \%$ of the patients. The mean and median of the disease period were 24.90 and 19 months, respectively, with a range of 2 to 108 months. The condition occurred most frequently in the cervical (47.50 \%) and lumbar $(25 \%)$ areas. Forty percent $(40 \%)$ of the patients developed syringomyelia.

1 Instituto Nacional de Enfermedades Neoplásicas, Departamento de Neurocirugía. Lima, Perú.

2 Hospital Nacional Arzobispo Loayza, Departamento de Neurocirugía. Lima, Perú.

3 Hospital Nacional Almanzor Aguinaga, Departamento de Neurocirugía. Chiclayo, Perú.

a Médico Neurocirujano, Doctor en Medicina.

b Médico Neurocirujana. 
Concerning their size, $60 \%$ of the tumors covered three to five levels, and $32.50 \%$ were larger than five levels. Thirty percent (30\%), $60 \%$ and $10 \%$ of the patients underwent a total, subtotal and partial resection, respectively. Seventyseven point five zero percent $(77.50 \%)$ of the patients had grade II ependymoma, $15 \%$ had grade I and $7.50 \%$ had grade III. Thirty-three (33) patients ( $82.50 \%)$ received radiotherapy (RTX) and three, chemotherapy (CTx). Overall survival accounted for $\mathbf{2 4 0}$ months. Age, sex, tumor size and disease were not statistically significant predictors of survival. Total resection showed survival rates of $100 \%, 90 \%$ and $90 \%$ at 5, 10 and 15 years, respectively, and statistically significant differences in relation to partial and subtotal resections $(p<0.011)$. There were statistically significant differences in the group of patients who received radiotherapy $(p<0.002)$. Progression-free survival accounted for 168 months. There were statistically significant differences in the progression-free survival regarding total resection and adjuvant radiotherapy, but no differences in the other variables. A mild to moderate motor deficit improved life prognosis during postoperative and follow-up periods.

Conclusions: Several factors affect overall survival and progression-free survival rates, with the most frequent being total resection and radiotherapy, and the least frequent a good preoperative motor function.

Keywords: Ependymoma; Intramedullary; Radiotherapy; Drug therapy; Prognosis (Source: MeSH NLM).

\section{INTRODUCCIÓN}

Los ependimomas son tumores que se originan de las células ependimarias del sistema ventricular y del canal central de la médula espinal. Son los más frecuentes de los tumores intramedulares, que son poco comunes; además, representan del 20 al $30 \%$ de los tumores espinales y aparecen, sobre todo, en la edad adulta ${ }^{(1)}$. La Organización Mundial de la Salus (OMS) los clasifica en tres grados: grado I (mixopapilar y subependimoma), grado II (celular, de células claras, papilar y tanacítico) y el ependimoma anaplásico (2). El ependimoma mixopapilar es benigno, no es infiltrativo $\mathrm{y}$, por lo general, proviene del complejo del cono medular, cola de caballo y filum terminale, en esta variante se puede realizar una resección total. El ependimoma grado II es de baja malignidad y el más frecuente, con un $55 \%$ al $75 \%$ del total de ependimomas; se ubica con mayor frecuencia a nivel cervical y su recurrencia es muy baja después de una resección total (3). El ependimoma anaplásico es infiltrativo dentro del parénquima medular y solo se puede tratar con una resección subtotal o parcial ${ }^{(4,5)}$. La característica histológica del ependimoma grado II es la presencia de seudorrosetas, en el $80 \%$ de casos ${ }^{(3)}$.

El tiempo de enfermedad es muy variable, de algunos meses a varios años. Los síntomas más frecuentes son disestesias y dolor, más adelante ya dependen de la localización y del compromiso de estructuras medulares, luego se agrega adormecimiento y, al final, sobreviene déficit motor en miembros inferiores o en los cuatro miembros ${ }^{\left({ }^{6}\right)}$.

La resonancia magnética nuclear (RMN) ha permitido realizar un diagnóstico cada vez más temprano con sintomatología incipiente. Se observan lesiones hiperintensas en T2 y la recuperación de la inversión atenuada de fluido (FLAIR, de sus siglas en inglés, Fluid-Attenuated Inversion Recovery), y lesiones hipo o isointensas en T1, con captación de contraste homogéneo, en algunos casos con formación quística y/o siringomielia, principalmente, a nivel cervical ${ }^{(7,8)}$.

En la antigüedad, los ependimomas fueron asociados con una elevada morbilidad quirúrgica en comparación con otros tumores espinales. Con la primera publicación en 1907 de un ependimoma intramedular operado con éxito se inicia la era de la cirugía oncológica medular ${ }^{(9)}$.

En la actualidad, sabemos que la resección quirúrgica es la mejor alternativa para mejorar el estado funcional y la sobrevida del paciente y que ha evolucionado para obtener una menor morbilidad postquirúrgica. La mayoría de cirujanos en el mundo está de acuerdo en que los ependimomas intramedulares son tumores bien delimitados del tejido de la médula espinal. Aunque la mayoría de los ependimomas están bien delimitados del tejido medular, la resección total sigue representando un desafío, como lo indican varios estudios que muestran una morbilidad quirúrgica mayor en comparación a otros tumores intramedulares. Se conoce que los pacientes que ingresan a operarse solo con disestesias (McCormick grado I) o con leve disminución de fuerza (McCormick grado II) son los que tienen mejor pronóstico de vida a largo plazo ${ }^{(9)}$.

La sobrevida global (SG) y la sobrevida libre de progresión (SLP) han mejorado significativamente en los últimos 30 años con la ayuda de la resonancia magnética, del microscopio quirúrgico de mejor resolución, el uso del monitoreo electrofisiológico intraoperatorio de la médula espinal, una mayor experiencia del cirujano y los pacientes que ingresaron con McCormick grado I ${ }^{(5)}$. Abdullah reporta una sobrevida global de $98 \%$ a los 5 años; de $87 \%$ a los 10; $y$ de $53 \%$ a los $15^{(10)}$.

Este artículo presenta un análisis detallado de 40 pacientes con ependimoma intramedular que fueron operados entre los años 1985 y 2017 en el Instituto Nacional de Enfermedades Neoplásicas (INEN) para identificar los 
factores pronósticos que mejoraron el estado funcional y la sobrevida del paciente, asociados al tratamiento complementario con radioterapia y quimioterapia.

La supervivencia global (SG) y la supervivencia libre de progresión de enfermedad (SLP) de los pacientes sometidos a resección tumoral de ependimomas intramedulares han sido reportados a nivel internacional desde hace varias décadas ${ }^{(11-13)}$. Sin embargo, en nuestro país la incidencia es incierta, por lo que se considera importante establecer una base de datos epidemiológicos y verificar la función neurológica del paciente intervenido, contrastando su evolución desde la evaluación inicial hasta su recuperación y rehabilitación posterior a la cirugía y al tratamiento complementario.

\section{MATERIALES Y MÉTODOS}

\section{Diseño y población de estudio}

Estudio observacional, descriptivo y retrospectivo. De un total de 192 pacientes operados de tumores espinales en el departamento de Neurocirugía del INEN en 33 años (enero de 1985 a diciembre de 2017), 40 pacientes, quienes furon tema del presente estudio, correspondieron a ependimoma de la médula espinal.

\section{Variables y mediciones}

Las variables fueron la edad, sexo, tiempo de enfermedad, grado de déficit neurológico preoperatorio, tamaño tumoral, tipo de resección quirúrgica, anatomía patológica, radioterapia y quimioterapia. Para las mediciones del cuadro neurológico se usó la escala modificada de McCormick (14).

\section{Escala modificada de McCormick}

Grado I: Neurológicamente normal, con mínima disestesia, marcha normal.

Grado II: Déficit sensitivo motor moderado, presencia de disestesias, es independiente.

Grado III: Déficit motor sensitivo moderado, requiere muletas para deambular, puede o no ser independiente.

Grado IV: Severo déficit sensitivo-motor, no deambula solo, es dependiente.

Grado V: Paraplejia o cuadriplejia.

\section{Análisis estadístico}

Se usó el programa estadístico SPSS versión 22 para Windows
(Statiscal Package for the Social Science); para el cálculo de la sobrevida se usó el estadístico del producto de los límites de Kaplan Meier, y para la comparación de medias y medianas de sobrevida empleamos el test del logaritmo del rango (Log Rank). La probabilidad (valor para ser considerado estadísticamente significativo) fue $p<0,05^{(15)}$.

\section{Consideraciones éticas}

Al tratarse de un estudio retrospectivo, que viene desde el año 1985, no existen contraindicaciones a la ética.

\section{RESULTADOS}

Entre los años 1985 y 2017 fueron operados 192 pacientes con tumores espinales en el Departamento de Neurocirugía del INEN, los más frecuentes fueron los de localización extramedular intradural (schwannomas). Pero los tumores intramedulares más frecuentes correspondieron a los ependimomas, extraídos de 40 pacientes $(20,83 \%)$ (Tabla 1$)$.

Tabla 1. Tumores espinales - INEN. 1985-2017: 192 casos

\begin{tabular}{|lcc|}
\hline Anatomía patológica & N. ${ }^{\circ}$ casos & Porcentaje (\%) \\
\hline Schwannomas & 70 & 36,45 \\
\hline Ependimomas & 40 & 20,83 \\
\hline Meningiomas & 17 & 8,85 \\
\hline Neurofibromas & 6 & 3,12 \\
\hline Otros tumores & 59 & 30,72 \\
\hline
\end{tabular}

De los 40 pacientes, 33 pacientes fueron adultos $(82,50 \%)$ y 7 , niños (17,50 \%). La edad promedio fue 34 años. El 57,50 \% de los casos eran del sexo masculino (23 pacientes) y el $42,50 \%$ fueron mujeres (17 casos), la razón ente ambos sexos fue $M: F=1,4: 1$.

Los síntomas al ingreso fueron déficit motor en 39 casos $(97,50 \%)$, dolor en 20 pacientes (50\%), disestesia $(42,50 \%)$, hipoestesia $(35,00 \%)$ y alteración de esfínteres $(27,50 \%)$.

La media y mediana del tiempo de enfermedad fue de 24,93 y 19 meses, respectivamente, con un rango de 2-108 meses. Los signos en el preoperatorio se midieron con la escala modificada de McCormick y mostraron que solo un paciente $(2,50 \%)$ correspondió a grado I, el $40,00 \%$ correspondió al grado II; 35,00\%, al grado III; y el 22,50\% fue del grado IV.

\section{Hallazgos radiológicos y en resonancia magnética} Del año 1985 a 1994 se usó como medio diagnóstico la mielografía con lopamidol como sustancia de contraste, y en los casos con bloqueo completo del canal espinal 
se amplió el estudio mielográfico con una punción suboccipitoatloidea. A partir de 1995, en todos los pacientes se usó resonancia magnética.

En nuestra serie, el $47,50 \%$ de los ependimomas se encontró a nivel cervical; el 25,00 \%, en la región lumbar; $15,00 \%$, en la región dorsal; y $12,50 \%$, en la región del cono medular y cola de caballo. La siringomielia se observó en 16 casos (40,00\%). El tamaño céfalo caudal del tumor se midió de acuerdo a niveles vertebrales. El porcentaje más alto correspondió a tumores que ocuparon de 3-5 niveles (24 pacientes), y también uno de cada tres pacientes se presentaron con tumores que ocupaban más de cinco niveles (Tabla 2 ).
Tabla 2. Ependimoma intramedular: 40 casos en INEN

\begin{tabular}{|lcc|}
\hline Localización & N. ${ }^{\circ}$ casos & Porcentaje \\
\hline Cervical & 19 & 47,50 \\
\hline Lumbar & 10 & 25,00 \\
\hline Dorsal & 6 & 15,00 \\
\hline Cono-cola de caballo & 5 & 12,50 \\
\hline Siringomielia & 16 & 40,00 \\
\hline Tamaño & & \\
\hline De 3 - 5 niveles & 24 & 60,00 \\
\hline Mayor de 3 niveles & 13 & 32,50 \\
\hline Menor de 3 niveles & 3 & 7,50 \\
\hline
\end{tabular}

La figura 1 muestra imágenes de RM de columna vertebral con contraste que describen al ependimoma grado I, ependimoma grado II y al ependimoma anaplásico.

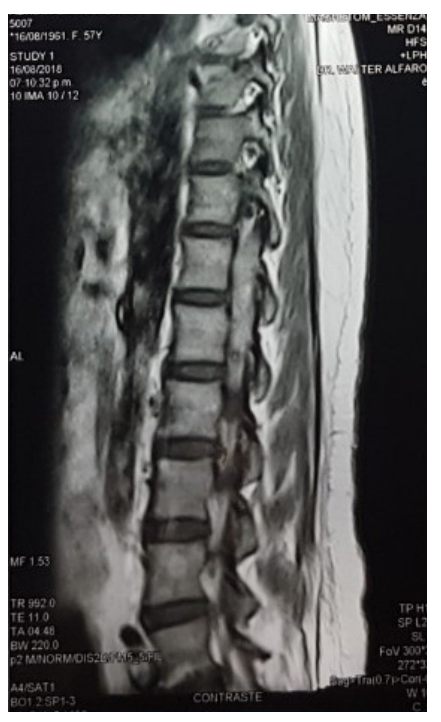

A. Hradiol

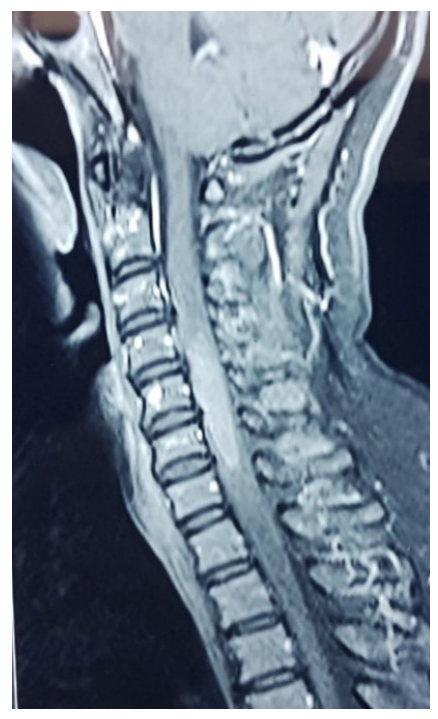

B. Hrailo II

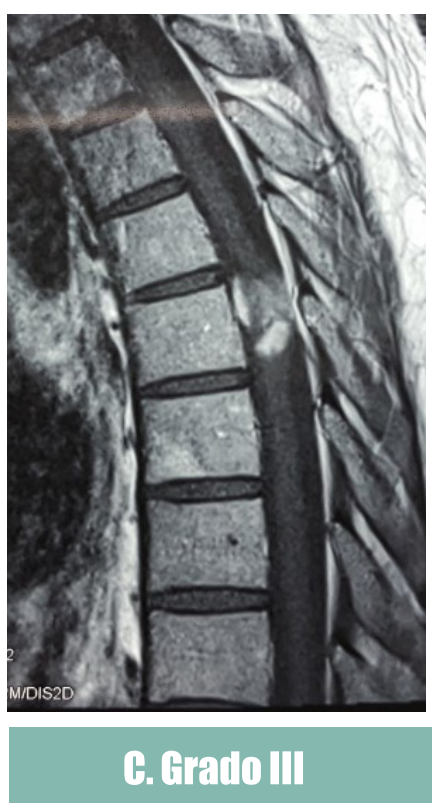

Figura 1. Ependimoma: grados I, II y III (OMS) .RM de columna vertebral con contraste: A. Ependimoma grado I, tumor localizado en el nivel D12-L1 de borde bien delimitado, ubicado en el cono medular; B. Ependimoma grado II, localizado a nivel C4 a C7, captación homogénea de contraste, y C. Ependimoma grado III, anaplásico, localizado a nivel D8, captación difusa de contraste.

\section{Complicaciones}

En el postoperatorio los pacientes presentaron las siguientes complicaciones: infección de herida operatoria en 3 casos (7,50\%), fístula de líquido cefalorraquídeo en 2 pacientes $(5,00 \%), 2$ casos de infección urinaria $(5,00 \%)$ y 1 paciente con hematoma epidural (2,50\%).

\section{Anatomía patológica}

En 31 pacientes $(77,50 \%)$ la histopatología correspondió a ependimoma grado II, el $15,00 \%$ fueron tumores mixopapilares y tres pacientes tuvieron ependimomas anaplásicos.

\section{Tratamiento complementario}

A 33 pacientes $(82,50 \%)$ se les administró radioterapia (RT) en el posoperatorio, en cantidad de 5400 a 6000 cGys (200 cGys por día), la RT fue bien tolerada. La quimioterapia se administró en tres pacientes $(7,50 \%)$ con ependimoma 
anaplásico, la droga usada fue temozolamida, en seis cursos (un curso por mes), y no se presentaron efectos secundarios.

Estado funcional en el preoperatorio $y$ en el postoperatorio del corto plazo

De acuerdo a la escala de McCormick modificada, en el preoperatorio ingresó solo un paciente con grado I, mientras que el 40,00\% de casos fue de grado II; 35,00\%, de grado III; y $22,50 \%$, con grado IV.
El deterioro neurológico transitorio en el posoperatorio fue evidente en la mitad de casos (50,00\%). El síndrome dolor neuropático después de la operación se observó en 16 pacientes $(40,00 \%)$.

La mediana de sobrevida global fue 240 meses. La mediana de sobrevida global a 5, 10 y 15 años fue de $84,40 \%, 81,20$ $\%$ y $63,70 \%$, respectivamente (Figura 2 ).

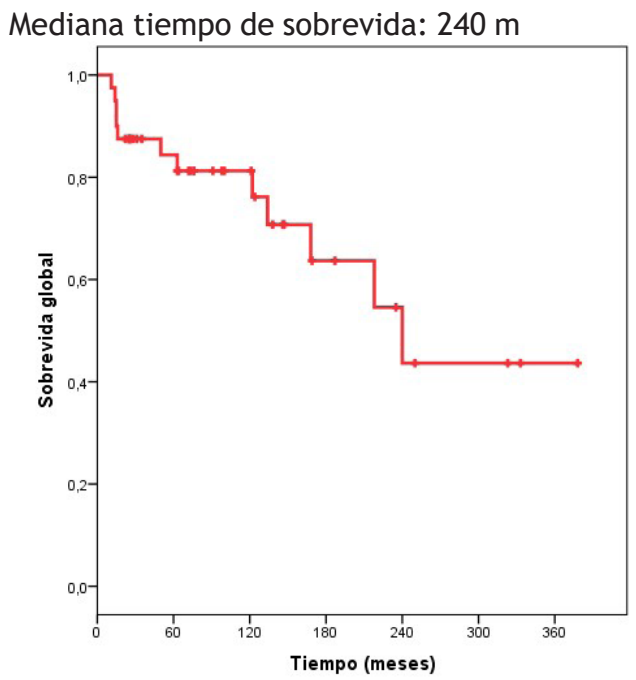

Figura 2. Mediana de sobrevida global . Ependimoma intramedular ( 40 casos) INEN

\begin{tabular}{|cccc} 
& \multicolumn{3}{c}{ Mediana de sobrevida según la edad } \\
& $60 \mathrm{~m}$ & $120 \mathrm{~m}$ & $180 \mathrm{~m}$ \\
\hline Niños & $85,70 \%$ & $85,70 \%$ & $57,10 \%$ \\
\hline Adultos & $84,10 \%$ & $80,20 \%$ & $65,40 \%$ \\
\hline
\end{tabular}

Cuando se realizó la comparación de las curvas de sobrevida según el sexo, la edad, el tamaño del tumor y la histopatología no encontramos diferencias estadísticamente significativas.
En el presente trabajo encontramos diferencias estadísticamente significativas cuando se realizó resección total $(p<0,011)$, y las medianas de sobrevida global cuando se realizó resección total, subtotal y parcial a 5, 10 y 15 años (Figura 3). 


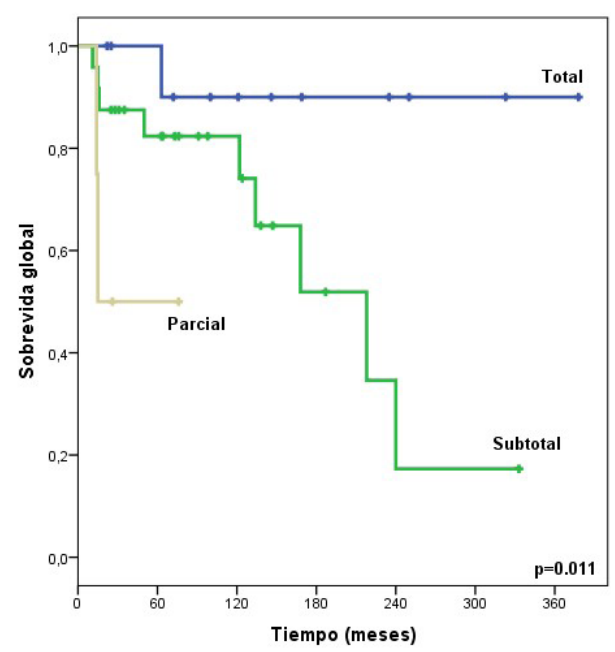

Figura 3. Sobrevida global: tipo de resección. Ependimoma intramedular(40 casos) INEN

\begin{tabular}{|c|c|c|c|}
\hline \multicolumn{4}{|c|}{ Mediana de sobrevida según el tipo de resección } \\
\hline & $60 \mathrm{~m}$ & $120 \mathrm{~m}$ & $180 \mathrm{~m}$ \\
\hline Total & $100,00 \%$ & $90,00 \%$ & $90,00 \%$ \\
\hline Subtotal & $82,40 \%$ & $82,40 \%$ & $51,90 \%$ \\
\hline Parcial & $50,00 \%$ & - & - \\
\hline
\end{tabular}

También encontramos que existieron diferencias estadísticamente significativas cuando se comparó el grupo de pacientes que sí recibió radioterapia $(p<0,002)$. En la figura 4 se muestran las medianas de sobrevida global a 5,10 y 15 años para ambos grupos.

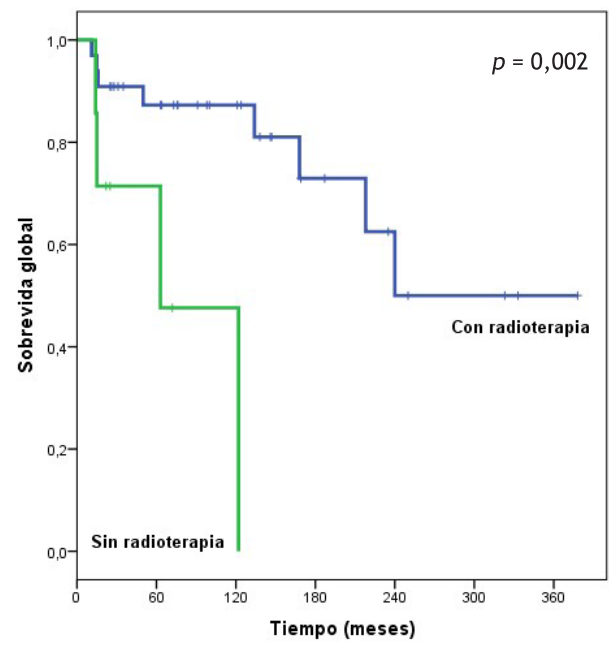

Figura 4. Sobrevida global: radioterapia. Ependimoma intramedular (40 casos) INEN 


\begin{tabular}{|cccc|}
\hline & \multicolumn{3}{c}{ Mediana de sobrevida según radioterapia } \\
& $60 \mathrm{~m}$ & $120 \mathrm{~m}$ & $180 \mathrm{~m}$ \\
\hline Con RT & $87,30 \%$ & $87,30 \%$ & $72,90 \%$ \\
\hline Sin RT & $71,4 \%$ & $47,6 \%$ & - \\
\hline
\end{tabular}

La mediana del tiempo de sobrevida libre de progresión (SLP) fue de 168 meses; la sobrevida libre de recurrencia a 5, 10 y 15 años fue de $76,00 \%, 65,90 \%$ y $46,00 \%$, respectivamente. La figura 5 muestra que hubo diferencias estadísticamente significativas en la SLP según el grado de resección quirúrgica y radioterapia adyuvante. Cuando se compararon las curvas de sobrevida libre de progresión en relación a la edad, sexo, tamaño tumoral, tipo de resección quirúrgica e histopatología, tampoco se observaron diferencias estadísticamente significativas.

Además, nuestro análisis evidenció que los pacientes con un buen estado clínico funcional preoperatorio (grado 1 y 2 de McCormick) tuvieron mejor pronóstico a largo plazo.

Mediana del tiempo de sobrevida: $168 \mathrm{~m}$

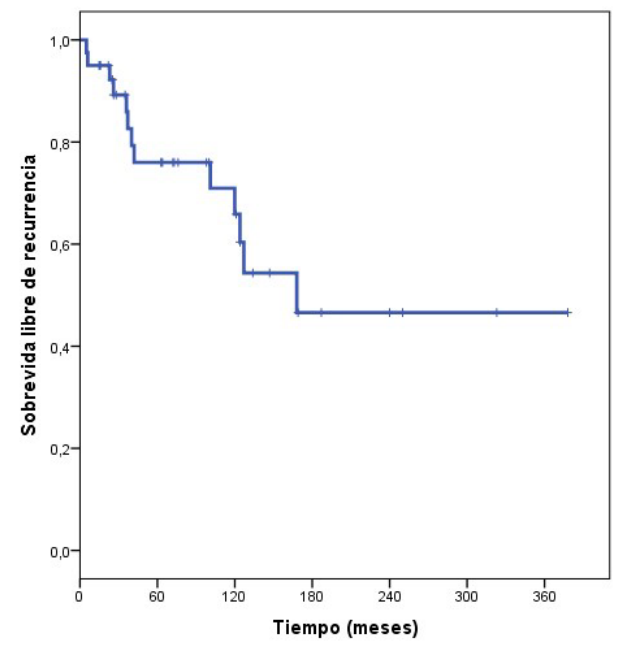

Figura 5. Sobrevida: Libre de recurrencia.Ependimoma intramedular (40 casos) INEN

\begin{tabular}{|ccc}
\hline \multicolumn{3}{c}{ Mediana de sobrevida: Libre de recurrencia } \\
\hline $60 m$ & $120 m$ & $180 m$ \\
\hline $76,00 \%$ & $65,90 \%$ & $46,60 \%$ \\
\hline
\end{tabular}

\section{Resultados del estado funcional a largo plazo}

El análisis del estado funcional se realizó en el seguimiento y en el último control mediante la escala modificada de McCormick. El único paciente que ingresó con McCormick grado I se mantuvo en similar grado. De 16 pacientes que ingresaron con McCormick grado II, seis mejoraron a grado I y cuatro se mantuvieron en el mismo grado. Por el contrario, los otros cuatro pacientes avanzaron a grados III, IV y V. De 14 enfermos que ingresaron con McCormick grado III, tres mejoraron a grado I y siete, a grado II; los otros cuatro pacientes se mantuvieron o avanzaron a grado IV y V. Finalmente, de los 9 pacientes que ingresaron con grado IV, solo dos casos mejoraron a grado III y los otros 7 se mantuvieron 
en ese grado o avanzaron a grado V. En la supervivencia global influyó la resección quirúrgica total, seguida de radioterapia adyuvante. Además, en nuestra serie se evidenció que los pacientes con un buen estado clínico funcional preoperatorio (grado 1 y 2 de McCormick) tuvieron mejor pronóstico a largo plazo (Tabla 3).

Tabla 3. Escala de McCormick preoperatoria / último control

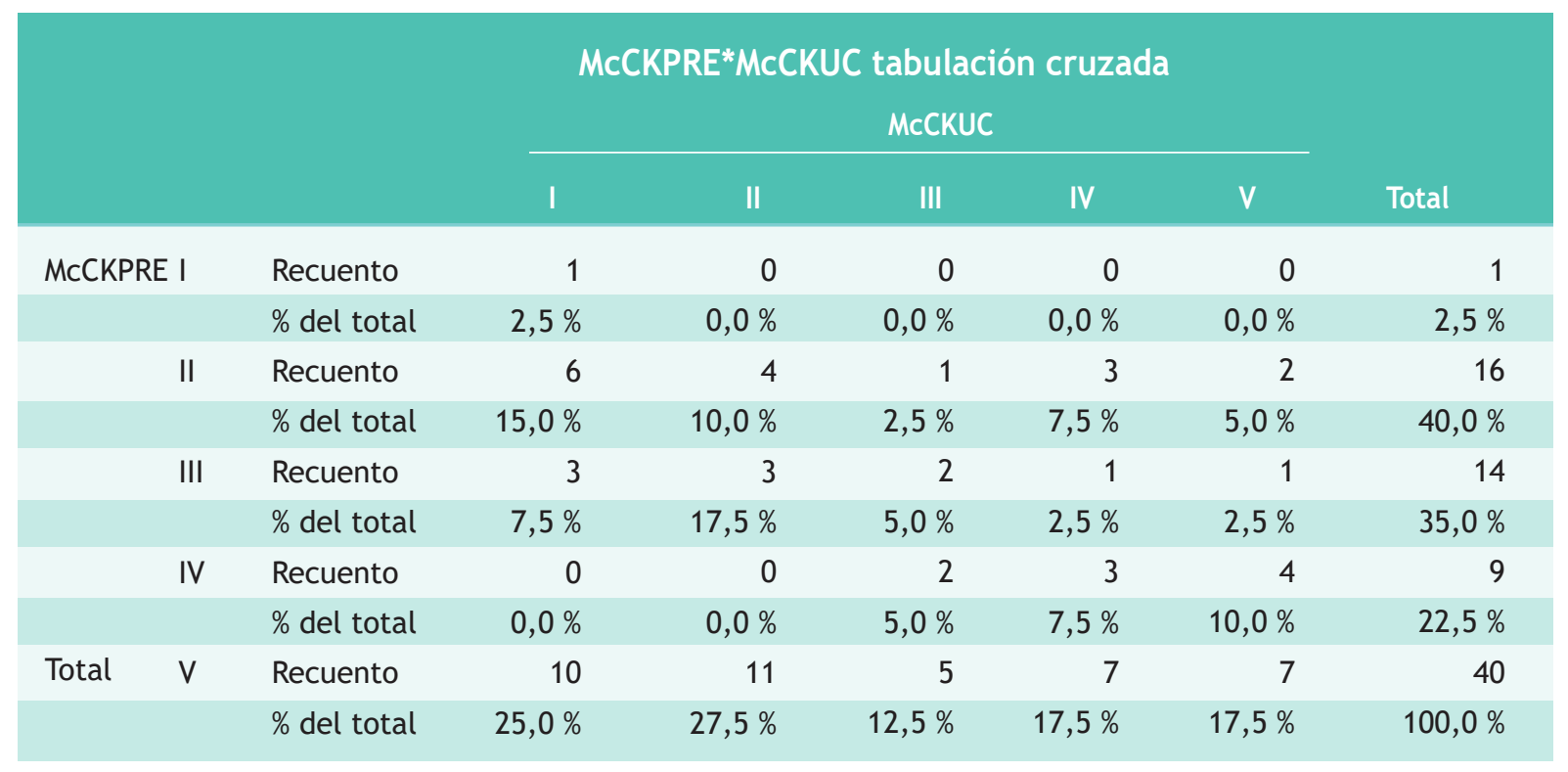

\section{DISCUSIÓN}

Los ependimomas intramedulares son mucho más frecuentes en adultos, y la distribución por sexo es casi similar, con una leve predominancia en hombres. Estos tumores son de crecimiento lento y muestran un curso relativamente benigno. Predominan entre la tercera y cuarta década de vida ${ }^{(2,3,10)}$. Por lo general, los síntomas más frecuentes son disestesias y dolor, el déficit motor se agrega posteriormente. La duración de los síntomas es muy variable, puede ser de algunos meses a varios años $(9,16,17)$.

En nuestra serie de 40 casos, los adultos representan el $82,50 \%$, la edad promedio fue 34 años, y la relación hombres: mujeres fue de 1,4:1. Los síntomas más frecuentes fueron la disminución de fuerza en el $97,50 \%$ y dolor en la mitad de casos; la media del tiempo de enfermedad fue de 24,9 meses. Encontramos que 39 pacientes acudieron a la primera consulta con algún grado de déficit motor en miembros inferiores o en los cuatro miembros. El $40,00 \%$ acudió a la consulta con un tumor McCormick grado II; y 35,00 \%, con grado II. Nosotros empezamos con mielografía como medio diagnóstico desde el año 1985 hasta 1994, y a partir del año 1995 con resonancia magnética. La RMN ha permitido, desde su introducción, un mejor abordaje a esta enfermedad, con un diagnóstico más temprano en los grados más bajos de McCormick, una mejor definición de la lesión, lo que lleva a una planificación quirúrgica individualizada, así mismo, el acceso actual a esta herramienta permite un adecuado seguimiento postquirúrgico y la detección temprana de una recurrencia. Encontramos que la localización más frecuente fue a nivel cervical en el 47,50 \%, y la menos frecuente, en el complejo cono medular y cola de caballo $(12,50 \%)$. En relación al tamaño tumoral, el $60,00 \%$ de pacientes fue de $3-5$ niveles, y el $32,00 \%$, mayor de 5 niveles; estos datos coinciden con todas las publicaciones revisadas $(2,3,5,9,10,16)$.

Con relación al tamaño tumoral, el $60,00 \%$ y 32,00 \% de pacientes fue de 3-5 niveles y mayor de 5 niveles respectivamente; estos datos coinciden con todas las publicaciones revisadas $(2,3,5,9,10,16)$.

En la mayoría de los trabajos publicados, el mayor porcentaje de pacientes ingresan a operarse con McCormick grado I y logran una mejor sobrevida global y sobrevida libre de progresión después de una resección total $(5,9,10)$.

Klekamp (9) reporta 100 casos, a partir de 1991, de los que el 79,00\% se clasificaron como McCormick grado I mediante el estudio de RMN en el preoperatorio. En la serie de 53 pacientes adultos de Abdullah (10), el 89,00\% acudió con 
alteración sensitiva y el $62,00 \%$ con algún grado de déficit motor. Sbovoda ${ }^{(5)}$, en su publicación de 37 pacientes adultos, reporta disestesias en el $67,00 \%$ y algún grado de déficit motor en el $44,00 \%$ de casos. En nuestra serie, el mayor porcentaje de pacientes acuden a la consulta con tumores de gran tamaño: en el 60,00\% medían de 3-5 niveles $y$ en el $32,50 \%$ fue mayor de 5 niveles. Solo un paciente $(2,50 \%)$ ingresó a operarse con diagnóstico de McCormick grado I; el $40,00 \%$, con grado II; $35,00 \%$ con un grado III, y $22,50 \%$ con McCormick grado IV. Además, se realizó la resección total en el 30,00\% de casos; subtotal, en el $60,00 \%$, y parcial, en el $10,00 \%$.

En nuestra serie, los seis ependimomas mixopapilares estuvieron localizados a nivel D12-L2. Como tratamiento aplicamos la resección total y obtuvimos una mejoría, a la normalidad, en el postoperatorio y con fisioterapia. La mayoría de series revisadas muestran casuística de ependimomas grado ll y anaplásicos, y algunas publicaciones incluyen al ependimoma mixopapilar. Gelabert-Gonzales ${ }^{(16)}$ reporta 20 casos consecutivos de ependimoma mixopapilar con resección total y con una mejoría total de la función motora-sensitiva y esfinteriana.

En esta investigación, siete pacientes fueron niños que ingresaron con algún grado de déficit motor; en tres de ellos se realizó una resección total, con mejoría lenta y progresiva de la función motora-sensitiva. Mc Girt et al. (17) evaluaron una población pediátrica con tumores de la médula espinal, que incluyeron a los ependimomas intramedulares. Estos pacientes acudieron a la consulta con disestesias y leve déficit motor; los resultados del postoperatorio fueron satisfactorios en casi la totalidad de casos, con recuperación completa de la función motora.

La Asociación Europea de Neuro-Oncología (EANO) publicó en Noviembre de 2017 las Guías para el Diagnóstico y Tratamiento de los Tumores Ependimarios del Sistema Nervioso Central. En ellas se aclara que en los ependimomas intramedulares el tratamiento de elección siempre es la resección total del tumor, y que la radioterapia solo debe ser administrada para los ependimomas si la resección es incompleta ${ }^{(18)}$.

El astrocitoma intramedular muestra bordes difusos en la $\mathrm{RMN}$, y se puede distinguir de un ependimoma porque este presenta una lesión de bordes bien delimitados, puede tener una formación quística (brillante en T2) y, en la mitad de los casos, se acompaña de siringomielia ${ }^{(6-8,19)}$.

La resección quirúrgica total sigue siendo el estándar en el tratamiento de los ependimomas intramedulares $y$, actualmente, puede ser alcanzada en casi el $80,00 \%$ de los casos, según datos citados por la mayoría de autores (20-25). Gracias al continuo avance tecnológico es posible contar con microscopios de última generación, aspiración ultrasónica, monitoreo intraoperatorio con potenciales evocados y tener el número suficiente de camas en la unidad de cuidados intensivos para un adecuado control postoperatorio del patrón respiratorio, ya que que el mayor porcentaje de estos tumores se localizan a nivel cervical (26-34). Nuestro centro hospitalario cuenta con estas herramientas desde hace 15 años, por lo que el porcentaje de resección total fue en el 30,00 \% de casos.

En 2015, Klekamp (9) publica una serie de casos de 100 pacientes con ependimoma intramedular. El 86,30\% fue sometido a una resección total, y alcanzaron una SG de 15 años y con SLP. El trabajo de Oh et al. ${ }^{(33)}$ comunica que la SG y la SLP a 15 años es del 100,00\% en pacientes operados con resección total sin RT comparado con los pacientes sometidos resección subtotal sin y con RT, en los que la SG y SLP es el 70,00 \% y 65,00\%, respectivamente.

Las complicaciones más frecuentes en el postoperatorio reportadas por varios autores están relacionadas con infección de herida operatoria y fístula de LCR ${ }^{(5,30-34)}$. En nuestra serie tuvimos ocho pacientes $(20,00 \%)$ con complicaciones en el postoperatorio, todos mejoraron con manejo médico y no fue necesario reingresar a sala de operaciones.

Sun (1) recomienda la RT solo para pacientes con resección incompleta y para los ependimomas con recurrencia de enfermedad. Una revisión (33) de 68 artículos con 348 casos de ependimoma intramedular señala que la RT fue administrada solo a los pacientes con resección incompleta que mejoraron la función motriz y la SLP; los autores concuerdan con que la RT no debe administrarse en pacientes sometidos a resección total en ependimoma grado II. Otros autores coinciden en este tipo de protocolo ${ }^{(1,4,12,35-37)}$.

Dulai et al. (38) comunican un caso de ependimoma intramedular con diseminación al cerebro. En nuestro estudio, no observamos ningún paciente con diseminación leptomeníngea.

El uso de la quimioterapia es controversial. Chamberlain et al. ${ }^{(39)}$ administran QT en casos de ependimoma anaplásico recurrente y usan etopósido, pero consideran que este tratamiento tiene un valor muy limitado. Las guías de la EANO (18) refieren que podría usarse la QT en pacientes con recurrencia tumoral y proponen el uso de etopósido o bevacizumab.

En nuestra serie, tres pacientes menores de 14 años operados por ependimoma anaplásico de localización dorsal que se trató con resección incompleta recibieron QT con temozolamida (6 cursos) después de la RT.

Los factores de buen pronóstico para todas las series fueron la resección total del tumor y, en los casos de resección 
incompleta o recurrencia tumoral, la RT. En pocas series publicadas comentaron que se administró QT en los casos de recurrencia tumoral.

En nuestra serie, los factores de buen pronóstico que influyeron en la sobrevida global fueron la resección total (con una sobrevida a 5, 10 y 15 años de 100,00\%, $90,00 \%$ y $90,00 \%$, respectivamente) y la radioterapia (sobrevida en el grupo de pacientes que recibió RT de $87,30 \%, 87,30 \%$ y $72,90 \%$, respectivamente). Estos dos factores también contribuyeron en la sobrevida libre de progresión. En este análisis también se confirmó que los pacientes que ingresaron con un McCormick grado I o II tuvieron una mejoría significativa en el seguimiento a largo plazo.

Contribuciones de los autores: José Enrique Orrego Puelles contribuyó en la elaboración de las secciones introducción, materiales y métodos, resultados y discusión. Katya Meryluz Chávez Barboza contribuyó en la recolección de datos de las historias clínicas, en los resultados y discusión. Mary Sylvia Emily Heredia Estela contribuyó en la recolección de datos, resultados y discusión.

Fuentes de financiamiento: El artículo ha sido financiado por los autores.

Conflicto de interés: Los autores declaran no tener ningún conflicto de interés.

\section{REFERENCIAS BIBLIOGRÁFICAS}

1. Sun $X-Y$, Wang $W$, Zhang $T-T$, Kong $C$, Sun $S-Y$, Guo $M-C$, et al. Factors associated with postoperative outcomes in patients with intramedullary Grade II ependymoma. A Systematic review and meta-analysis. Medicine (Baltimore). 2019; 98(25): e16185.

2. Louis DN, Ohgaki H, Wiestler OD, Cavenee WK, Burger PC, Jouvet A, et al. The 2007 WHO classification of tumours of the Central Nervous System. Acta Neuropathol. 2007; 114(2): 97-109.

3. Louis DN, Ohgaki H, Wiestler OD, Cavenee WK. WHO Classification of Tumours of the Central Nervous System. 4th ed. IARC: Lyon; 2016.

4. Celano E, Salehani A, Malcolm JG, Reinertsen E, Hadjipanayis CG. Spinal cord ependymoma: a review of the literature and case series of ten patients. J Neurooncol. 2016; 128(3): 377-86.

5. Svoboda N, Bradac 0 , de Lacy P, Benes V. Intramedullary ependymoma: long-term outcome after surgery. Acta Neurochir (Wien). 2018; 160(3): 439-47.

6. Engelhard HH, Villano JL, Porter KR, Stewart AK, Barua M, Barker FG, et al. Clinical presentation histology and treatment in 430 patients with primary tumors of the spinal cords, spinal meninges, or cauda equina. J Neurosurg Spine. 2010; 13(1): 67-77.

7. Yuh EL, Barkovich AJ, Gupta N. Imaging of ependymomas: MRI and CT. Childs Nerv Syst. 2009; 25(10): 1203-13.

8. Klekamp J, Samii M. Syringomyelia, Diagnosis and Treatment. Berlín: Springer; 2001.

9. Klekamp J. Spinal ependymomas. Part I: Intramedullary ependymomas. Neurosurg Focus. 2015; 39 (2): E6.

10. Abdullah KG, Lubelski D, Miller J, Steinmetz MP, Shin JH, Krishnaney $A$, et al. Progression free survival and functional outcome after surgical resection of intramedullary ependimomas. J Clin Neurosci. 2015; 22(12): 1933-7.

11. Malis LI. Intramedullary spinal tumors cord. Clin Neurosurg. 1978; 25: 512-39.

12. Peschell RE, Kapp DS, Cardinale F, Manuelidis EE. Ependymoma of the spinal cords. Int J Radiotherapy Oncol Biol Phys. 1983; 9(7): 1093-6.

13. Shaw EG, Evans RG, Sceithauer BW, Ilstrup DM, Earle JD. Radiotherapeutic management of adults intraspinal ependymoma. J Radiother Oncol Biol Phys. 1986; 12(3): 323-7.

14. McCormick PC, Torres R, Post KD, Stein BM. Intramedullary ependymoma of the spinal cord. J Neurosurg. 1990; 72(4): 523-32.

15. Kaplan EL, Meier P. Nonparametric estimation from incomplete observations. J Am Stat Assoc. 1958; 53(282): 457-81.

16. Gelabert-González M, Arcos-Algaba A, Serramito-García R, CastroBouzas D, Santín-Amo JM, Aran-Echabe E, et al. Ependimomas del filum terminal. Análisis de 20 casos consecutivos. Neurocirugía. 2010; 21(5): 381-9.

17. Mc Girt MJ, Chaichana KL, Atiba A, Attenello F, Yao KC, Jello GI. Resection of intramedullary spinal cords tumors in children: assessmet of long-term motor and sensory deficits. J Neurosurg Pediatr. 2008; 1(1): 63-7.

18. Rudà R, Reifenberger G, Frappaz D, Pfister SM, Laprie A, Santarius T, et al. EANO guidelines for the diagnosis and treatment of ependymal tumors. Neuro Oncol. 2018; 20(4): 445-56.

19. Eroes CA, Zausinger S, Kreth F-W, Goldbrunner R, Tonn J-C. Intramedullary low grade astrocytoma and ependymoma. Surgical results and predicting factors for clinical outcome. Acta Neurochir (Wien). 2010; 152(4): 611-8.

20. Wostrack $M$, Ringel $F$, Eicker SO, Jägersberg $M$, Schaller $K$, Kerschbaumer J, et al. Spinal ependymoma in adults: a multicenter investigation of surgical outcome and progression-free survival. J Neurosurg Spine. 2018; 28(6): 654-62.

21. Boström A, Von Lehe M, Hartmann W, Pietsch T, Feuss M, Boström JP, et al. Surgery for spinal cord ependymomas: outcome and prognostic factors. Neurosurgery. 2011; 68(2): 302-9.

22. Garcés-Ambrossi GL, Mc Girt MJ, Mehta VA, Sciubba DM, Witham TF, Bydon A, et al. Factors associated whit progression-free survival and long-term neurological outcome after resection of intramedullary spinal cord tumors: analysis of 101 consecutive cases. J Neurosurg Spine. 2009; 11(5): 591-9.

23. Ebner FH, Roser F, Falk M, Hermann S, Honegger J, Tatagiba M. Management of intramedullary spinal cord lesions: interdependence of the longitudinal extension of the lesion and the functional outcome. Eur Spine J. 2010; 19(4): 665-9.

24. Vera-Bolaños E, Aldape K, Yuan Y, Wu J, Wani K, Necesito-Reyes MJ, et al. Clinical course and progression-free survival of adults intracranial and spinal ependymomas patients. Neuro Oncol. 2015; 173(3): 440-7.

25. Adam Y, Benezech J, Blanquet A, Fuentes J-M, Bousigue J-Y, Debono $B$, et al. Intramedullary tumors. Results of a national investigation in private neurosurgery. Neurochirurgie. 2010; 56(4): 344-9.

26. Aghakhani N, David P, Parker F, Lacroix C, Benoudiva F, Tadie M. Intramedullary spinal ependymoma: analisys of consecutive series of 82 adult cases with particular attention to patients with no preoperative neurological deficit. Neurosurg. 2008; 62(6): 1279-85.

27. Kucia EJ, Bambakidis NC, Chang SW, Spetzler RF. Surgical technique and outcomes in the treatment and spinal cord ependymoma, part 1: intramedullary ependymomas. Neurosurgery. 2011; 68(Suppl. 1): 57-63.

28. Lee S-M, Cho Y-E, Kwon Y-M. Neurological outcome after surgical treatment of intramedullary spinal cords tumors. Korean J Spine. 2014; 11(3): 121-6.

29. Li T-Y, Chu J-S, Xu Y-L, Yang J, Wang J, Huang Y-H, et al. Surgical 
strategies and outcomes of spinal ependymomas of different lengths: analysis of 210 patients. J Neurosurg Spine. 2014; 21(2): 249-59.

30. Manzano G, Green BA, Vanni S, Levi AD. Contemporary management of adult pathology and neurological outcome related to surgical resection. Spinal Cord. 2008; 46(8): 540-6.

31. Sun $X-Y$, Kong C, Lu S-B, Sun S-Y, Guo M-C, Ding J-Z. Survival outcomes and prognostic factors of patients with intramedullary Grade II ependymomas after surgical treatments. J Clin Neurosci. 2018; 57: 136-42.

32. Hanbali F, Fourney DR, Marmor E, Suki D, Rhines LD, Weinberg JS, et al. Spinal cord ependymoma: radical surgery resection and outcome. Neurosurgery. 2002; 51(5): 1172-4.

33. Oh MC, Kim JM, Kaur G, Safaee M, Sun MZ, Singh A, et al. Prognosis by tumor location in adults with spinal ependymomas. J Neurosurg Spine. 2013; 18(3): 226-35.

34. Tobin MK, Geraghty JR, Engelhard HH, Linninger AA, Mehta Al. Intramedullary spinal cord tumors: a review of current and future treatment strategies. Neurosurg Focus. 2015; 39 (2): E14.

35. Lee S-H, Chung CK, Kim CH, Yoon SH, Hyun S-J, Kim K-J, et al. Long-term outcomes of surgical resection with or without adjuvant radiation therapy for treatment of spinal ependymoma: a retrospective multicenter study by the Korea Spinal Oncology Research Group. Neuro Oncol. 2013; 15(7): 921-9.

36. Clover LL, Hazuka MB, Kinzie JJ. Spinal cord ependymomas treated with surgery and radiation therapy. A review of 11 cases. Am J Clin Oncol. 1993; 16(4): 350-3.

37. Wen BC, Hussey DH, Hitchon PW, Schelper RL, Vigliotti AP, Doornbos $\mathrm{JF}$, et al. The role radiation therapy in the management of ependymomas of the spinal cord. Int J Radiat Oncol Biol Phys. 1991; 20(4): 781-6.

38. Dulai MS, Caccamo DV, Briley AL, Edwards MSB, Fisher PG, Lehman NL. Intramedullary papillary ependymoma with chordoid plexus differentiation and cerebrospinal fluid dissemination to the brain. J Neurosurg Pediatric. 2010; 5(5): 511-7.

39. Chamberlain MC. Salvage chemotherapy for recurrent spinal cord ependymoma. Cancer. 2002; 95(5): 997-1002

\section{Correspondencia:}

Enrique Orrego Puelles

Dirección: Av. Trinidad Morán 1210 - Lince. Lima, Perú. Teléfono: 998709289

Correo electrónico: eorregopuelles@hotmail.com

Recibido: 18 de noviembre de 2020

Evaluado: 19 de enero de 2021.

Aprobado: 19 de febrero de 2021 .

( ) La revista. Publicado por Universidad de San Martín de Porres, Perú. (cc) Br Licencia de Creative Commons Artículo en acceso abierto bajo términos de Licencia Creative Commons Atribución 4.0 Internacional. (http://creativecommons.org/licenses/by/4.0/)

ORCID iDs

José Enrique Orrego Puelles $\odot$ https: / / orcid.org/0000-0002-0979-3997

Katya Meryluz Chávez Barboza ㄷttps://orcid.org/0000-0002-6788-3376

Mary Sylvia Emily Heredia Estela ㄴ https://orcid.org/0000-0001-8674-0031 